\title{
O ÍNDICE IBOVESPA E A LÓGICA FUZZY: UMA NOVA ESTRATÉGIA DE INVESTIMENTO
}

\author{
IBOVESPA index and fuzzy logic: a new investment strategy
}

\author{
Cesar Duarte Souto-Maior \\ Mestre pelo CPGA/UFSC. Florianópolis, SC - Brasil. \\ e-mail: cesarcdm@yahoo.com.br \\ Fernando Dal-Ri Murcia \\ Doutorando da FEA/USP. São Paulo, SP - Brasil. \\ e-mail: fernandomurcia@hotmail.com \\ José Alonso Borba \\ Professor do CPGA/UFSC. Florianópolis, SC - Brasil. \\ e-mail jalonso@cse.ufsc.br \\ Newton C. A. da Costa Jr. \\ Professor do CPGA/UFSC. Florianópolis, SC - Brasil. \\ e-mail: newton@cse.ufsc.br
}

\section{Resumo}

Este trabalho apresenta uma nova aplicação da lógica fuzzy: a previsão da direção do movimento do índice IBOVESPA. Para testar essa abordagem, foram selecionadas as variações diárias do índice da Bolsa de Valores de São Paulo (IBOVESPA) durante o período de janeiro de 1997 a fevereiro de 2005, perfazendo um total de 2.000 observações. A primeira metade das observações foi usada como estimação e a segunda metade para previsão. Embora o modelo estimado gere uma saída lingüística, foi possível delinear uma estratégia de investimento estatisticamente significante, que supera a rentabilidade da estratégia buy-and-hold. Para aplicações futuras, esta proposta pode ser incrementada com o uso de outras informações econômicas e não-econômicas, inclusive com a intuição, para auxiliar as decisões de investimento e produzir previsões melhores.

Palavras-chave: Lógica fuzzy; Previsão; Índice BOVESPA.

\section{Abstract}

This paper presents a new application of fuzzy logic: forecasting the direction/sign of the change in price levels. In order to test this approach, the movements of the Sao 
Paulo Stock Exchange Index (IBOVESPA) were selected. The study period extends over 2,000 daily observations of the index, from January/1997 to February/2005. The first half of the observations was used for the estimation, while the second half was used for forecasting. Despite the fact that the estimated model produced a linguistic output, it was possible to delineate a statistically significant investment strategy, which outperformed a buy-and-hold one. For futures applications this proposal could be enhanced through the use of other economic and non economic information, inclusive intuition, to help investment decisions and to produce even better forecasts.

Keywords: Fuzzy logic; Forecasting; IBOVESPA Index.

\section{INTRODUÇÃO}

Desde a década de 1980 a literatura relacionada com séries financeiras temporais tem produzido importantes estudos que têm questionado as hipóteses de eficiência de mercado fraca e o random walk (RW), como, por exemplo, os trabalhos de Lo e Mackinley (1988), Poterba e Summers (1988), Fama e French (1988), entre outros. Esses autores argumentam que existe considerável evidência de que o retorno das ações é de alguma forma previsível. Eles mostraram que há fortes evidências de heteroscedasticidade condicional em muitas séries de tempo financeiras, significando que os retornos dessas séries não são independentes e identicamente distribuídos como estabelece o modelo RW.

Esses fatos têm despertado interesses teóricos e práticos em modelos não lineares de séries financeiras temporais baseadas em técnicas como auto-regressive moving average (família ARMA), generalized auto-regressive condicional heteroskedasticity (família GARCH), e mais recentemente, métodos baseados em inteligência artificial como as redes neurais artificiais (ANN), os algoritmos genéticos e a lógica fuzzy.

Basicamente, a previsão de séries financeiras temporais pode ser vista de duas formas: modelos de estimação do nível e modelos de previsão de direção. O primeiro se baseia na previsão acurada do nível de preços das ações, índices e outros instrumentos de séries financeiras. $\mathrm{O}$ grau de acurácia e aceitabilidade da previsão são medidos pelo seu desvio das observações reais, minimizando os erros de previsão. Exemplos dessa abordagem são dados por Teixeira e Rodrigues (1997), Pérez-Rodrigues, Torra e Andrada-Félix (2005) e Ribeiro e Silva (2005), que comparam diversos métodos baseados em inteligência artificial com o RW e outros métodos lineares, concluindo que 
os métodos de ANN apresentam melhor desempenho. Alguns métodos comumente usados para previsão do nível de séries financeiras temporais são a adaptative exponential smoothing, a multivariate transfer function, o Filtro de Kalman e a multilayered feedforward neural network.

A segunda maneira é a previsão da direção ou sinal da mudança do nível de preço. Essa abordagem é defendida por alguns autores (WU e ZHANG, 1997; AGGARWAL; DEMASKEY, 1997; TSAIH; HSU; LAI, 1998; LEUNG; DAOUK; CHEN, 2000; CHEN; LEUNG; DAOUK, 2003; KIN; CHUN, 1998) que argumentam que uma estratégia de negociação baseada em certa previsão com um pequeno erro de previsão pode não ser tão rentável quanto uma estratégia de negociação baseada em uma acurada previsão da direção ou sinal de movimento. Algumas técnicas comumente usadas como modelos de classificação são: a análise de discriminante, os modelos logit e probit, e as redes neurais probabilísticas.

Em sintonia com esse último ponto de vista, este estudo sugere o uso de uma nova abordagem com o intuito de prever a direção da mudança no nível de preço: a lógica fuzzy. Diferentemente de Huarng e Yu $(2005,2006)$ e Yu (2005), que também utilizam a lógica fuzzy, o modelo proposto não retorna um valor exato, e sim uma saída probabilística de variáveis lingüísticas. Essa saída lingüística pode ser utilizada com outras informações econômicas e não econômicas, inclusive com a intuição, para auxiliar a decisão de investimento.

É importante ressaltar que o presente modelo não pretende refutar ou ser comparado com outros modelos paramétricos ou não paramétricos, mas propor uma nova solução, baseada nos conceitos da lógica fuzzy. Este artigo é organizado da seguinte maneira. Na sessão 2 é apresentada uma breve introdução da lógica fuzzy e de seus conceitos. A sessão 3 apresenta a construção do modelo proposto. A sessão 4 apresenta os resultados finais e a sessão 5 discute e conclui o trabalho.

\section{Lógica Fuzzy}

Na lógica convencional (binária), inicialmente desenvolvida pelo filósofo grego Aristóteles (384 - 322 A.C.), uma proposição ou é verdadeira ou é falsa. Essa lógica assume que o estado natural dos eventos seja totalmente definido. Entretanto, na grande maioria dos problemas gerenciais, os estados dos eventos são vagos, e as transições entre o pertencer e o não pertencer não são totalmente definidas. 
Zadeh (1965) publicou o primeiro artigo sobre lógica fuzzy intitulado "Fuzzy Sets". O modelo foi desenvolvido para converter valores subjetivos em valores objetivos. Um conjunto fuzzy (nebuloso) não possui um limite preciso. A diferença entre pertencer e não pertencer é fuzzy e, portanto, há uma graduação de pertinência. De acordo com Zebda (1998, p. 27), “A teoria dos conjuntos fuzzy não é uma teoria de decisão, mas sim um cálculo (uma linguagem de modelagem) onde fenômenos vagos nos sistemas humanísticos podem ser tratados de forma sistemática".

Em um conjunto nebuloso, o grau de pertinência associado a cada elemento define o quanto cada objeto do universo do discurso satisfaz a propriedade associada ao conjunto.

Bojadziev e Bojadziev (1997, p. 9) definem um conjunto nebuloso como um conjunto de pares ordenados, uma relação binária da forma:

$$
\mathrm{A}^{\prime}=\{(x, \mu a(x)) \mid x \in \mathrm{A}, \mu a(x) \in[0,1]\}
$$

onde $\mu a(x)$ é chamada de função de pertinência e especifica o grau no qual cada elemento $x$ em A pertence ao conjunto nebuloso $\mathrm{A}^{\prime}$.

Para von Altrock (1997), a teoria dos conjuntos nebulosos é uma generalização que engloba os conjuntos convencionais quando $\mu a(x)=0$ ou $\mu a(x)=1$. Em outras palavras, os conjuntos convencionais são casos especiais dos conjuntos nebulosos.

O quadro 1 e a figura 1 ilustram as diferenças entre os conjuntos clássicos e os conjuntos nebulosos.

Quadro 1. Conjuntos Clássicos X Conjuntos Nebulosos.

\begin{tabular}{|l|l|}
\hline Conjuntos Clássicos - Lógica Binária & Conjuntos Nebulosos - Lógica Fuzzy \\
\hline Limites precisos & Limites Imprecisos \\
\hline Transição brusca entre pertencer e não pertencer & Transição gradual entre pertencer e não pertencer \\
\hline Representa conceitos bem definidos & Representam conceitos vagos e imprecisos \\
\hline
\end{tabular}


A

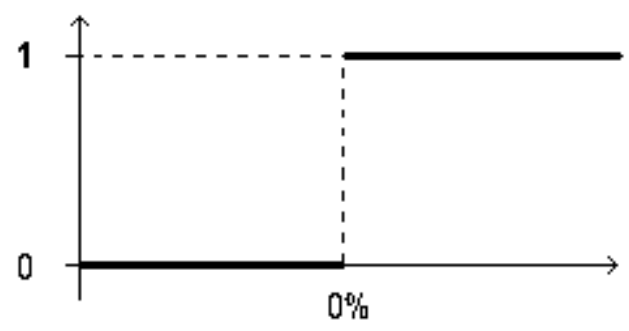

B

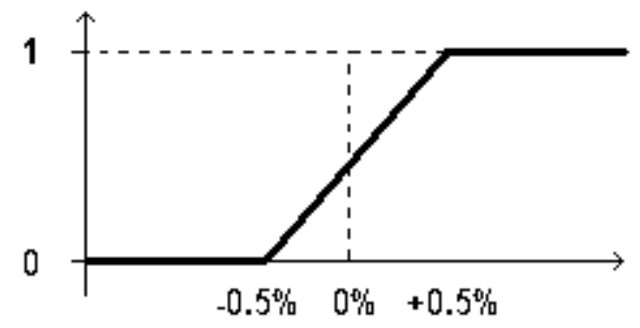

Figura 1. Conjuntos Clássicos X Conjuntos Nebulosos.

A figura 1 exemplifica um caso típico. No gráfico A, é utilizada a lógica clássica. Se a variação do índice IBOVESPA é positiva, a variação é considerada como "subida" e se for negativa, a variação é considerada como "queda". A transição entre pertencer e não pertencer é abrupta. Valores "infinitesimalmente" negativos são classificados como "queda" e valores "infinitesimalmente" positivos são classificados como "subida". Porém, o mercado pode considerar esses dois valores como a mesma informação e, dessa forma, um sistema baseado na lógica clássica pode apresentar um desempenho insatisfatório. O gráfico B mostra como a lógica fuzzy pode ser utilizada provendo uma transição suave entre "queda" e "subida".

\section{METODOLOGIA}

Na figura 2, temos uma visão geral do modelo desenvolvido, que basicamente está dividido em três partes principais: a fuzzificação das variáveis de entrada, as regras de inferências e as variáveis de saída. Aqui, a fuzzificação (defuzificação) é entendida como a conversão de valores numéricos (lingüísticos) em valores lingüísticos (numéricos).

\section{Modelo Fuzzy para Previsão do Indice IBOVESPA}

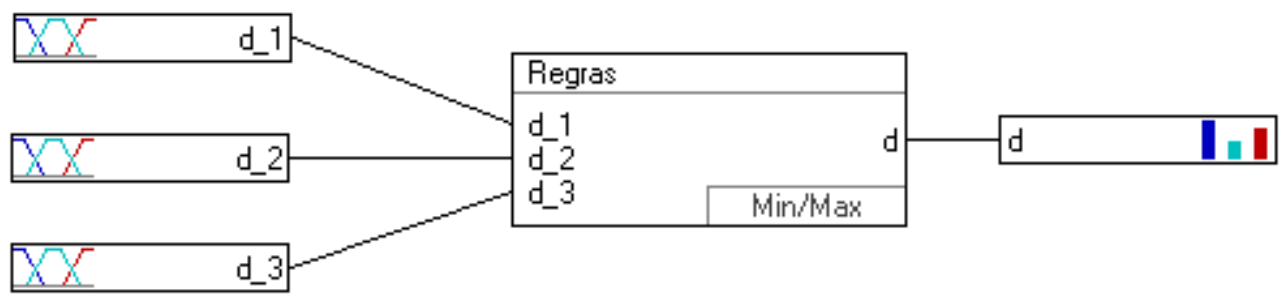

Figura 2. Visão Geral do Modelo. 
Onde d, d_1, d_2 e d_3 são as variações percentuais (em valores lingüísticos) do índice IBOVESPA nos dias d, d-1, d-2 e d-3, respectivamente.

Todavia, a construção do modelo conceitual foi dividida em quatro partes:

- escolha das variáveis para o modelo de previsão e definição do conjunto de dados para treinamento e teste;

- fuzzificação;

- regras de inferência;

- variáveis de saída;

Cada uma dessas etapas será descrita nas próximas sub-sessões.

\section{Estabelecimento das variáveis para o modelo de previsão e estabelecimento do conjunto de dados para treinamento e teste}

As variações percentuais do índice IBOVESPA nos três dias anteriores ao dia a ser previsto foram escolhidas como variáveis de entrada. Esta escolha está baseada no estudo de O’Connor, Remus e Griggs (1997). Eles mostram que indivíduos apresentam diferentes tendências e comportamentos para série de subidas e descidas. Assim, as pessoas podem tomar decisões com base em informações recentes. Por exemplo, se a bolsa apresentar alta durante vários dias consecutivos, poderá haver uma tendência à realização de lucros e com isso uma queda, mesmo que o cenário macroeconômico esteja favorável. Por outro lado, se a bolsa cair durante vários dias consecutivos, poderá haver uma tendência para que os preços voltem a subir, mesmo com um cenário macroeconômico desfavorável.

Ao todo foram coletados 2.000 conjuntos diários de dados, com a variação percentual do dia em questão e as variações percentuais dos três dias anteriores. Os dados foram coletados na Economática. O período analisado foi de 8 de janeiro de 1997 até 2 de fevereiro de 2005.

Para o cálculo da variação percentual no dia d foi utilizada a seguinte fórmula:

$$
\operatorname{Var}_{d}=\frac{\left(V_{d}-V_{d-1}\right)}{V_{d-1}}
$$


onde $\operatorname{Var}_{d}$ é a variação numérica do índice IBOVESPA no dia d, $V_{d}$ e $V_{d-1}$ são os valores do índice IBOVESPA no dia d e no dia d-1, respectivamente.

Os primeiros 1.000 conjuntos diários de dados foram utilizados para o treinamento e estabelecimento das regras do modelo, correspondendo ao período de 8 de janeiro de 1997 até 22 de janeiro de 2001. Os 1.000 conjuntos diários de dados seguintes foram utilizados para o teste do modelo, correspondendo ao período de 23 de janeiro de 2001 até 2 de fevereiro de 2005.

\section{Processo de fuzzificação}

Todas as variáveis numéricas de entrada do modelo fuzzy precisam ser convertidas em variáveis lingüísticas. Neste estudo, os valores lingüísticos de entrada adotados foram "queda" e "subida". Neste trabalho, "baixo" será considerado sinônimo de "queda", "médio" será considerado sinônimo de "estabilidade" e "alto" de "subida".

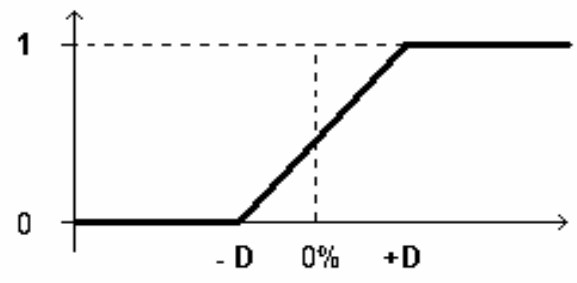

Figura 3. Gráfico da função de pertinência "subida” da variável lingüística d_1.

A Figura 3 mostra o gráfico da função de pertinência "subida" da variável lingüística d_1, que pode ser descrita da seguinte maneira:

$$
\mu s=\left\{\begin{array}{lll}
0, & \text { para } & \operatorname{Vaf}_{-1} \leq D \\
1 / 2+\operatorname{Vaf}_{-1} / 2 D, & \text { para } & -D<\operatorname{Vaf}_{-1}<+L \\
1, & \text { para } & \operatorname{Var}_{d_{1}} \geq+D
\end{array}\right.
$$

onde ${ }^{\mu s}$ é a função de pertinência "subida" e $V_{a r_{-1}}$ é a variação numérica do índice no dia d-1, ou seja, ao dia anterior. 


\section{Estabelecimento das regras de inferência}

Depois da fuzzificação de todas as variáveis de entrada, o próximo passo envolve o estabelecimento de regras de inferência. Essas regras representam uma das formas que a inteligência humana usa para tomar decisões, partindo de premissas lógicas. Para o problema em questão foram criadas 24 regras de inferência. Essas regras são declarações lógicas e, para cada regra, pode ser atribuído um valor de zero até um chamado Degree of Support (DoS), que depende de características do conjunto de treinamento. Quando uma regra possui o DoS igual a zero (um), a regra é considerada insignificante (significante). O DoS também admite valores entre zero e um para regras parcialmente significativas. Segue abaixo uma das regras utilizadas.

Se (d_3 = "subida" e d_2 = "subida" e d_1 = "subida")

Então $d=$ "subida", com um DoS (Degree of Support) de 0,44.

Para a atribuição das regras de inferências foram selecionados, dos 1.000 conjuntos de treinamento, 557 conjuntos característicos. Esses conjuntos característicos têm os valores de d_1 associados com 100\% "queda" ou 100\% "subida", os valores de d_2 associados com 100\% "queda" ou 100\% "subida", e os valores de d_3 associados com 100\% "queda" ou 100\% "subida". A tabela 1 mostra a distribuição desses conjuntos.

\begin{tabular}{|l|l|l|l|}
\hline d_3 & d_2 & d_1 & Conjuntos \\
\hline baixo & baixo & baixo & 71 \\
\hline baixo & baixo & alto & 74 \\
\hline baixo & alto & baixo & 58 \\
\hline baixo & alto & alto & 83 \\
\hline alto & baixo & baixo & 65 \\
\hline alto & baixo & alto & 56 \\
\hline alto & alto & baixo & 67 \\
\hline alto & alto & alto & 83 \\
\hline
\end{tabular}

Para uma verificação preliminar do modelo do modelo foram selecionados, dos 1.000 conjuntos de teste, 422 conjuntos característicos. Como anteriormente, esses conjuntos característicos têm os valores de d_1 associados com 100\% "queda" ou 100\% 
"subida", os valores de d_2 associados com 100\% "queda" ou 100\% "subida", e os valores de d_3 associados com 100\% "queda" ou 100\% "subida". A tabela 2 mostra a distribuição desses conjuntos.

\begin{tabular}{|l|l|l|l|}
\hline d_3 & d_2 & d_1 & Conjuntos \\
\hline baixo & baixo & baixo & 44 \\
\hline baixo & baixo & alto & 63 \\
\hline baixo & alto & baixo & 48 \\
\hline baixo & alto & alto & 61 \\
\hline alto & baixo & baixo & 50 \\
\hline alto & baixo & alto & 50 \\
\hline alto & alto & baixo & 50 \\
\hline alto & alto & alto & 56 \\
\hline
\end{tabular}

Tabela 2. Dados selecionados para teste.

A figura 4 apresenta a distribuição probabilística dos dados de treinamento e de teste quando a entrada é o conjunto (d_3 = queda; d_2 = subida; d_1 = queda). As probabilidades de queda e subida são bem parecidas. Nesse caso, o gestor de um fundo de investimento não teria informação adicional para auxiliar o processo decisório, apenas a incerteza inerente ao mercado.
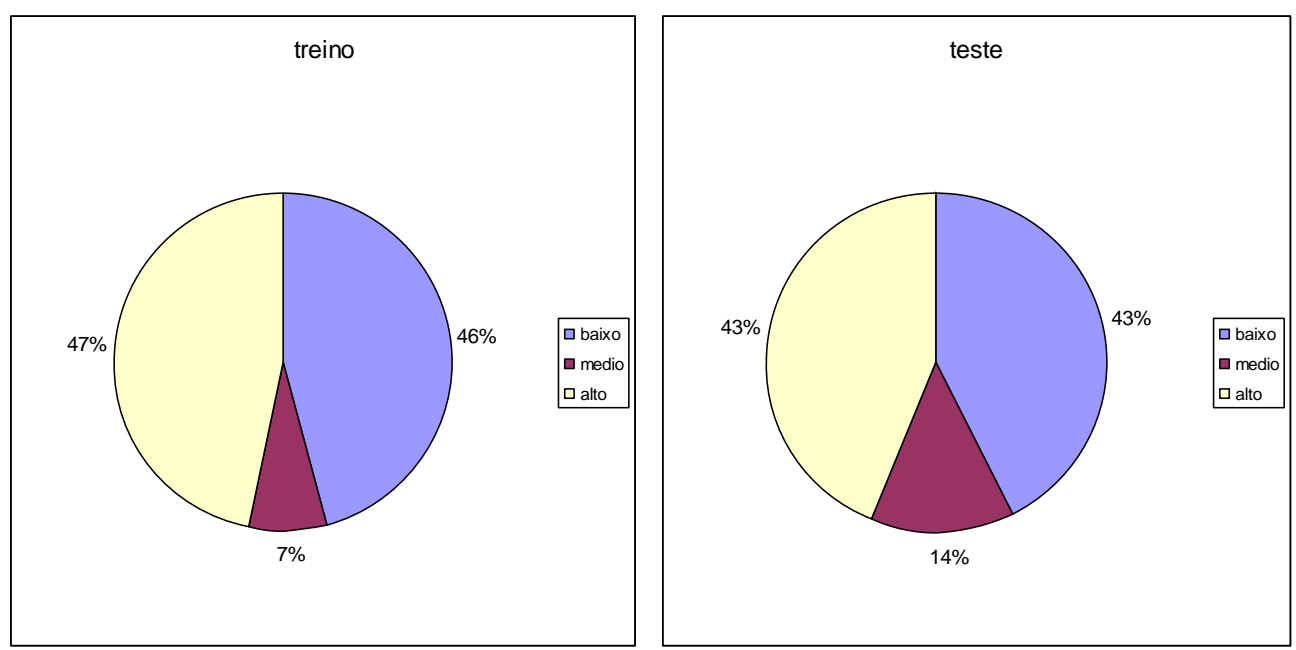

Figura 4. Distribuição probabilística dos dados de treinamento e teste quando a entrada é (d_3 = queda; d_2 = subida; d_1 = queda).

Já a figura 5 apresenta a distribuição probabilística dos dados de treinamento e de teste quando a entrada é o conjunto (d_3 = subida; $d \_2$ = queda; d_1 = subida). Nesse caso, a probabilidade de subida é maior do que a probabilidade de queda. O gestor de um fundo de investimento poderia utilizar essa informação para auxiliar o processo decisório. 
O Índice IBOVESPA e a Lógica Fuzzi: uma nova estratégia de investimento

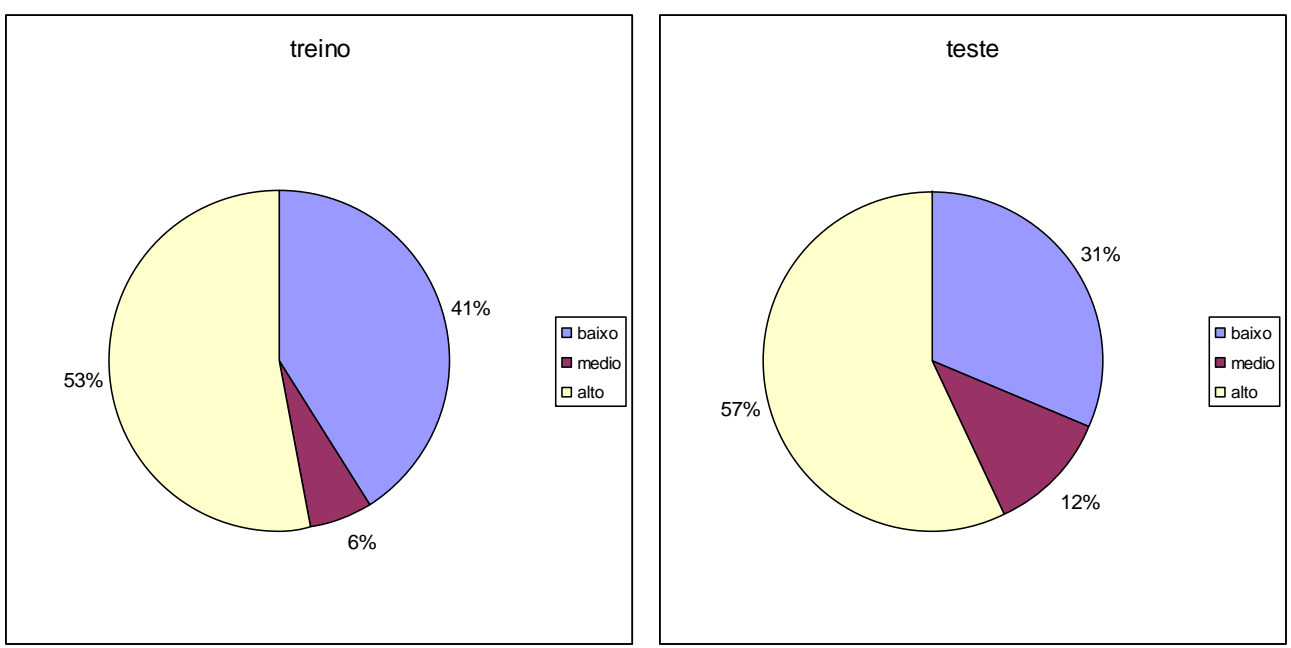

Figura 5. Distribuição probabilística dos dados de treinamento e teste quando a entrada é (d_3 = subida; d_2 = queda; d_1= subida).

Com base na distribuição probabilística de saída foram atribuídas as regras de inferência. A Tabela 3 apresenta as 24 regras utilizadas.

\begin{tabular}{|l|l|l|l|l|}
\hline \multicolumn{2}{|c|}{ IF } & \multicolumn{2}{|c|}{ THEN } \\
\hline d_1 & d_2 & d_3 & DoS & d \\
\hline Low & low & Low & 0.43 & low \\
\hline Low & low & Low & 0.06 & medium \\
\hline Low & low & Low & 0.51 & high \\
\hline Low & low & High & 0.45 & low \\
\hline Low & low & High & 0.04 & medium \\
\hline Low & low & High & 0.51 & high \\
\hline Low & high & low & 0.46 & low \\
\hline Low & high & low & 0.07 & medium \\
\hline Low & high & low & 0.47 & high \\
\hline Low & high & high & 0.38 & low \\
\hline Low & high & high & 0.14 & medium \\
\hline Low & high & high & 0.48 & high \\
\hline High & low & low & 0.45 & low \\
\hline High & low & low & 0.06 & medium \\
\hline High & low & low & 0.49 & high \\
\hline High & low & high & 0.41 & low \\
\hline High & low & high & 0.06 & medium \\
\hline High & low & high & 0.53 & high \\
\hline High & high & low & 0.53 & low \\
\hline High & high & low & 0.08 & medium \\
\hline High & high & low & 0.38 & high \\
\hline High & high & high & 0.42 & low \\
\hline High & high & high & 0.14 & medium \\
\hline High & high & high & 0.44 & high \\
\hline
\end{tabular}

Tabela 3. Regras de inferência. 


\section{Estabelecimento das variáveis de saída}

A saída do modelo será denominada $d$, que representa a variação do índice IBOVESPA no dia a ser previsto. Os valores lingüísticos de saída adotados foram: "queda", "estabilidade" e "subida". No presente estudo não foi necessário a defuzzificação. Os valores lingüísticos foram utilizados como variáveis de saída, representando a probabilidade de o índice apresentar um comportamento de queda, estabilidade ou subida.

A variável de saída $d$ pode ser representada como um vetor de dimensão $3 \times 1$ da seguinte forma:

$$
d=\left[\begin{array}{l}
d 1 \\
d 2 \\
d 3
\end{array}\right],
$$

onde $d 1$ é o componente lingüístico "queda" da saída $d, d 2$ é o componente lingüístico "estabilidade" da saída $d$ e $d 3$ é o componente lingüístico "subida" da saída $d$.

\section{RESULTADOS}

Após a construção do modelo, foi estabelecida uma estratégia para um fundo de investimentos fictício. Portanto, a estratégia adotada se resume a um fundo passivo baseado no índice IBOVESPA, porém nos dias em que o componente lingüístico "queda" da variável de saída $d$ for maior do que uma variável denominada $\varepsilon$, o dinheiro é retirado do fundo, sendo reinvestido no dia seguinte. Por exemplo, se $\varepsilon=0,5$ significa que o dinheiro será retirado do fundo quando a probabilidade de queda, indicada pelo modelo fuzzy, for maior que 50\%. Está implícita nessa estratégia a suposição que o fundo é capaz de comprar e vender ações na mesma proporção que o índice IBOVESPA.

Em suma, foi utilizado o seguinte algoritmo:

$$
\begin{gathered}
\text { Se }\left(d 1_{i}>\varepsilon\right) \\
\text { Então } \alpha_{i=0} \\
\text { Senão } \alpha_{i=1}
\end{gathered}
$$


Onde $d 1_{i}$ é o componente lingüístico "queda" para o dia i, $\alpha_{i}=1$ significa que o dinheiro deve ser aplicado no fundo no dia i e $\alpha_{i}=0$ significa que o dinheiro deve ser retirado do fundo no dia i.

Para a simulação do modelo foram adotadas as premissas de um "frictionless market". Em outras palavras, todos os custos e associados com as transações foram considerados inexistentes. Também não foram considerados os retornos que poderiam ser obtidos em títulos de renda fixa nos dias em que o dinheiro não é aplicado no fundo.

Para calcular o rendimento do fundo passivo, ou seja, a estratégia de buy-andhold, foi utilizada a seguinte fórmula:

$$
\prod_{i=1}^{1000}(1+\operatorname{Var} \& / 100)
$$

O valor resultante da equação acima é o retorno total do índice IBOVESPA durante o período de teste.

Para calcular o rendimento do fundo baseado na estratégia do modelo fuzzy, foi utilizada a seguinte fórmula:

$$
\prod_{i=1}^{1000}\left[\alpha_{i} \times\left(1+\operatorname{Var} d_{i} / 100\right)+\left(1-\alpha_{i}\right)\right]
$$

A estratégia foi simulada com a aplicação de vários valores de $\varepsilon$. A tabela 4 apresenta os resultados obtidos.

\begin{tabular}{|l|l|l|l|l|}
\hline \multicolumn{1}{|c|}{ Valores de $\boldsymbol{\varepsilon}$} & \multicolumn{1}{|c|}{ (a) Fuzzy } & \multicolumn{1}{|c|}{ (b) IBOVESPA } & diferença (a-b) & Índice de Sharpe \\
\hline 0,480 & $71,04 \%$ & $41,48 \%$ & $29,56 \%$ & 0,0393 \\
\hline 0,485 & $54,89 \%$ & $41,48 \%$ & $13,41 \%$ & 0,0336 \\
\hline 0,490 & $61,80 \%$ & $41,48 \%$ & $20,32 \%$ & 0,0360 \\
\hline 0,495 & $68,52 \%$ & $41,48 \%$ & $27,04 \%$ & 0,0382 \\
\hline 0,500 & $76,74 \%$ & $41,48 \%$ & $35,26 \%$ & 0,0408 \\
\hline 0,505 & $77,80 \%$ & $41,48 \%$ & $36,33 \% \quad(*)$ & 0,0409 \\
\hline 0,510 & $71,68 \%$ & $41,48 \%$ & $30,20 \%$ & 0,0389 \\
\hline 0,515 & $65,63 \%$ & $41,48 \%$ & $24,15 \%$ & 0,0368 \\
\hline 0,520 & $67,52 \%$ & $41,48 \%$ & $26,04 \%$ & 0,0374 \\
\hline 0,525 & $67,23 \%$ & $41,48 \%$ & $25,75 \%$ & 0,0372 \\
\hline 0,530 & $41,48 \%$ & $41,48 \%$ & $0,00 \%$ & 0,0278 \\
\hline
\end{tabular}

Tabela 4. Resultados obtidos com a estratégia fuzzy.

Nota: (*) indica significante ao nível de $10 \%$, teste unicaudal 
A estratégia passiva apresentou um rendimento de $41,48 \%$ no período de teste, ou seja, de 23 de janeiro de 2001 até 2 de fevereiro de 2005. Como pode ser observado, se o valor de $\varepsilon$ for maior ou igual a 0,53 , o rendimento será igual ao fundo passivo, pois o sistema fuzzy não retornou nenhum valor de probabilidade de queda superior a 0,53 para o conjunto de teste. Como pode ser observada na Tabela 4, a estratégia baseada no modelo fuzzy apresentou um rendimento de até 77,80\% no mesmo período. Em valores absolutos o modelo fuzzy superou o fundo passivo em até $36,33 \%$ com o valor da estatística t para o modelo unicaudal de 1,26, estatisticamente significante para o nível de $10 \%$.

O índice de Sharpe generalizado da estratégia passiva foi de 0,0278 e a Tabela 4 apresenta o índice de Sharpe generalizado (para cada valor de $\varepsilon$ ).

A Figura 6 mostra a rentabilidade de uma unidade monetária investida no fundo passivo e na estratégia fuzzy (para $\varepsilon=0,505$ ) durante o período de teste.

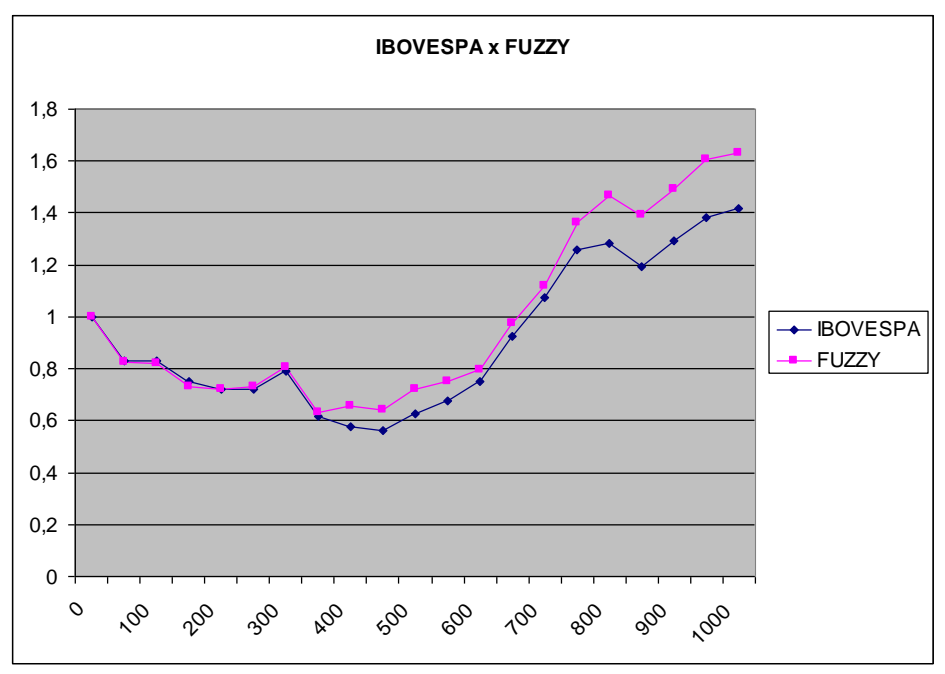

Figura 6. Rentabilidade do IBOVESPA e da estratégia fuzzy (para $\varepsilon=0,505$ ).

Também foi calculada a taxa de acerto de direção para os dias em que o dinheiro seria retirado do fundo para os vários valores de $\varepsilon$. A Tabela 5 apresenta os resultados obtidos. Como pode ser observado, foi alcançada uma taxa de acerto de até 55,42\%, para $\varepsilon=0,51$. Para esse valor de $\varepsilon$, o dinheiro teria sido retirado em 83 dias, e o índice teria apresentado um valor menor em 46 desses dias. Para $\varepsilon=0,480\left(\chi^{2}=277\right)$ o teste qui-quadrado foi estatisticamente significante para o nível de $10 \%$. 
O Índice IBOVESPA e a Lógica Fuzzi: uma nova estratégia de investimento

\begin{tabular}{|l|l|l|l|}
\hline \multicolumn{1}{|c|}{ Valores de $\varepsilon$} & \multicolumn{1}{c|}{ dias } & \multicolumn{1}{c|}{ acerto } & \multicolumn{1}{c|}{ taxa de acerto } \\
\hline 0,480 & 134 & 73 & $54,48 \% \quad(*)$ \\
\hline 0,485 & 127 & 67 & $52,76 \%$ \\
\hline 0,490 & 117 & 62 & $52,99 \%$ \\
\hline 0,495 & 110 & 59 & $53,64 \%$ \\
\hline 0,500 & 103 & 56 & $54,37 \%$ \\
\hline 0,505 & 90 & 49 & $54,44 \%$ \\
\hline 0,510 & 83 & 46 & $55,42 \%$ \\
\hline 0,515 & 72 & 39 & $54,17 \%$ \\
\hline 0,520 & 68 & 36 & $52,94 \%$ \\
\hline 0,525 & 61 & 33 & $54,10 \%$ \\
\hline 0,530 & 0 & 0 & Não aplicável \\
\hline
\end{tabular}

Tabela 5: Resultados obtidos com a estratégia fuzzy

Nota: (*) indica significante ao nível de $10 \%$, teste unicaudal

\section{CONCLUSÕES}

Este estudo apresenta um modelo baseado na lógica fuzzy para prever a direção de movimento do índice IBOVESPA. O período de estudo cobre 2.000 observações diárias do índice, de janeiro de 1997 até fevereiro de 2005. A primeira metade das observações é usada para a estimação e a segunda metade é usada para a previsão.

O modelo proposto retorna uma resposta não exata, com uma saída em termos probabilísticos. Porém, mesmo sendo imprecisa, a saída do modelo propicia a adoção de uma (estatisticamente significante) estratégia rentável de investimento que superou a estratégia de buy-and-hold durante o período de teste e na previsão da direção de sinal, foi obtido uma significância de $10 \%$ ao aplicar o teste qui-quadrado.

Além disso, o modelo proposto, com sua saída probabilística, pode ser utilizado como suporte a decisão, tendo em vista que o investidor pode possuir outras informações, confidenciais ou não, assim como pode ter até intuições a respeito de tendências políticas ou econômicas.

Há muitas possibilidades de novas pesquisas decorrentes deste trabalho. Uma delas é a escolha de novas variáveis de entrada e termos lingüísticos. Algo interessante seria testar o uso de mais do que três dias de variações anteriores. Por exemplo, testar para 4 e 5 variações anteriores. Além disso, testar o mesmo procedimento para previsão semanal e mensal pode dar bons resultados e reduzir os custos de transação. 
Pode ser feita a associação com anomalias consagradas como o efeito fim de semana e o efeito mês do ano, bem como com regras da análise técnica. Também pode ser feita a associação com as outras técnicas de inteligência artificial como as redes neurais e os algoritmos genéticos.

\section{REFERÊNCIAS}

AGGARWAL, R.; DEMASKEY, A. Using derivatives in major currencies for crosshedging currency risks in Asian emerging markets. Journal of Futures Markets, Hoboken, NJ, v. 17, p. 781-796, 1997.

BOJADZIEV, G.; BOJADZIEV, M. Fuzzy logic for business, finance and management. Singapore: World Scientific, 1997.

CHEN, A.; LEUNG, M. T.; DAOUK, H. Application of neural networks to an emerging financial market: forecasting and trading the Taiwan stock index. Computers \& Operational Research. Elsevier, v. 30, p. 901-923, 2003.

FAMA, E. F.; FRENCH, K. R. Permanent and temporary components of stock prices. Journal of Political Economics, Chicago, IL, v. 96, n. 2, p. 246-273, 1988.

HUARNG, K.; YU, H. A type 2 fuzzy time series model for stock index forecasting. Physica A, Boston, v. 353, p. 445-462, 2005.

HUARNG, K.; YU, H. Th application of neural networks to forecasting fuzzy time series. Physica A, Boston, v. 363, p. 481-491, 2006.

KIN, S. H.; CHUN, S. H. Graded forecasting using an array of bipolar predictions: application of probabilistic neural networks to a stock market index. International Journal of Forecasting, Australia, v. 14. p. 323-337, 1998.

LEUNG, M. T.; DAOUK, H.; CHEN, A. Forecasting stock indices: a comparison of classification and level estimation models. International Journal of Forecasting, Australia, v. 16, p. 173-190, 2000.

LO, A. W.; MACKINLAY, C. Stock market prices do not follow random walks: evidence from a simples specification test. Review of Financial Studies, Oxford, v. 1, n. 1, p. 41-66, 1988.

O’CONNOR, M.; REMUS, W.; GRIGGS, K. Going up-going down: how good are people at forecasting trends and changes in trends? Journal of Forecasting, Hoboken, NJ, v. 16, p. 165-176, 1997.

POTERBA, J.; SUMMERS, L. Mean reversion in stock prices: evidence and implications. Journal of Financial Economics, Rochester, NY, v. 22, n. 1, p. 27-59, 1988. 
PÉREZ-RODRÍGUES, J. V. P.; TORRA, S.; ANDRADA-FÉLIX, J. A. STAR ANN models: forecasting performance on the Spanish "IBEX-35" stock index. Journal of Empirical Finance. North-Holland, v. 12, p. 490-509, 2005.

TEIXEIRA, J. C.; RODRIGUES, A. J. An applied study on recursive estimation methods, neural networks and forecasting. European Journal of Operational Research, North-Holland, v. 101, p. 406-417, 1997.

TSAIH, R.; HSU, Y.; LAI, C. C. Forecasting S\&P 500 stock index futures with a hybrid AI system. Decision Support Systems, North-Holland, v. 23, p. 161-174, 1998.

VON ALTROCK, C. Fuzzy logic and neurofuzzy applications in business and finance. Upper Saddle River, New Jersey: Prentice Hall, 1997.

WU, Y.; ZHANG, H. Forward premius as unbiased predictors of future currency depreciation: a non-parametric analysis. Journal of International Money and Finance. Elsevier, v. 16, n. 4, p. 609-623, 1997.

YU, H. A refined fuzzy time-series for forecasting. Physica A, Boston, v. 346, p. 657$681,2005$.

ZADEH, L. F. S. Information and Control, Elsevier, v. 8, p. 338-353, 1965.

ZEBDA, A. The problem of ambiguity and the use of fuzzy set theory in accounting: a perspective and opportunities for research. Applications of fuzzy sets and the theory of evidence to accounting II, London, v. 7, p. 20-33, 1998.

Recebido:14/06/2007

Received: 06/14/2007

Aprovado: 10/08/2007

Aproveid: 08/10/2007 